This item was submitted to Loughborough's Research Repository by the author.

Items in Figshare are protected by copyright, with all rights reserved, unless otherwise indicated.

\title{
Comparable neutrophil responses for arm exercise and intensity-matched leg exercise
}

PLEASE CITE THE PUBLISHED VERSION

http://dx.doi.org/10.1249/MSS.0000000000001258

\section{PUBLISHER}

(c) American College of Sports Medicine (ACSM)

\section{VERSION}

AM (Accepted Manuscript)

\section{PUBLISHER STATEMENT}

This work is made available according to the conditions of the Creative Commons Attribution-NonCommercialNoDerivatives 4.0 International (CC BY-NC-ND 4.0) licence. Full details of this licence are available at: https://creativecommons.org/licenses/by-nc-nd/4.0/

\section{LICENCE}

CC BY-NC-ND 4.0

\section{REPOSITORY RECORD}

Leicht, Christof A., Victoria L. Goosey-Tolfrey, and Nicolette Bishop. 2019. "Comparable Neutrophil Responses for Arm Exercise and Intensity-matched Leg Exercise”. figshare.

https://hdl.handle.net/2134/24526. 
1 Comparable neutrophil responses for arm and intensity-matched leg exercise

2

3 Christof A. Leicht ${ }^{1}$, Victoria L. Goosey-Tolfrey ${ }^{1}$, Nicolette C. Bishop ${ }^{1}$

4

$5{ }^{1}$ The Peter Harrison Centre for Disability Sport; School of Sport, Exercise, and Health

6 Sciences; Loughborough University; Loughborough; UK

7

8 Corresponding author:

9 Dr Nicolette C. Bishop; School of Sport, Exercise, and Health Sciences; Loughborough

10 University; Loughborough LE11 3TU; UK

11 E-mail: n.c.bishop@lboro.ac.uk

12 Tel: +44 1509226385

13 Fax: +44 1509226301

14

15 Short title

16 Arm vs leg exercise: Neutrophil responses

17

18 
ABSTRACT

Introduction: Arm exercise is performed at lower absolute intensities than lower body exercise. This may impact on intensity dependent neutrophil responses and it is unknown whether individuals restricted to arm exercise experience the same changes in the neutrophil response as found for lower body exercise. Therefore, we aimed to investigate the importance of exercise modality and relative exercise intensity on the neutrophil response.

Methods: Twelve moderately trained males performed three 45-min constant load exercise trials following determination of peak oxygen uptake for arm exercise ( $\left.\dot{\mathrm{V}} \mathrm{O}_{2 \text { peak arms }}\right)$ and cycling ( $\left(\mathrm{VO}_{2 \text { peak legs }}\right)$ : (1) arm cranking exercise at $60 \% \dot{\mathrm{V}} \mathrm{O}_{2 \text { peak arms; }}$ (2) moderate cycling at

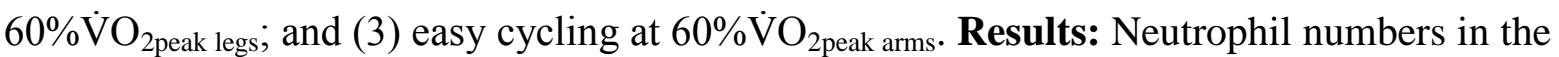
circulation increased for all exercise trials, but were significantly lower for easy cycling when compared with arm exercise $(\mathrm{P}=0.009)$, mirroring the blunted increase in heart rate and epinephrine during easy cycling. For all trials, exercising heart rate explained some of the variation of the neutrophil number $2 \mathrm{~h}$ post exercise $(\mathrm{R}=0.51-0.69)$, epinephrine explaining less of this variation $(\mathrm{R}=0.21-0.34)$. The number of neutrophils expressing CXCR2 decreased in the recovery from exercise in all trials $(\mathrm{P}<0.05)$. Conclusion: Arm and leg exercise elicits the same neutrophil response when performed at the same relative intensity, implying that populations restricted to arm exercise might achieve a similar exercise induced neutrophil response as those performing lower body exercise. A likely explanation for this is the higher sympathetic activation and cardiac output for arm and relative intensity matched leg exercise when compared with easy cycling, which is partly reflected in heart rate. This study further shows that the downregulation of CXCR2 may be implicated in exercise-induced neutrophilia.

Key words: upper body exercise, first line of defence, elastase, CXCR2, PSGL-1, IL-8, neutrophil degranulation 


\section{INTRODUCTION}

46 Engaging in a moderate amount/intensity of exercise is associated with a lower infection risk when compared with doing no exercise or very high amounts/intensities of exercise (19). This relationship and acute exercise immune responses have been investigated extensively for lower body exercise modalities. However, a limited amount of studies have focussed on populations restricted to upper body exercise $(11,13)$, which includes the 1.2 million wheelchair users in the UK (18) and the 7.4 million people in the US dependent on wheelchairs or assistive devices to support their mobility (3). Furthermore, direct comparisons of upper vs lower body exercise with respect to immune function are scarce and have so far been limited to cytokine and monocyte responses $(10,14)$. From a theoretical perspective, this modality comparison is intriguing as arm exercise is usually performed at lower absolute intensities than lower body exercise, which may impact on intensity dependent acute immune responses. Practically, this comparison is relevant for those restricted to the upper body exercise modality, for example for those with a disability or undergoing rehabilitation.

The body of research on lower body exercise modalities such as running or cycling is in support of acute moderate exercise as a means to alter blood borne markers of immunity this includes cytokine secretion and the leukocyte activation state associated with improved host defence $(14,22,24)$. Further, neutrophil numbers increase following exercise with a peak at around 2-4 hours post exercise $(19,28,31)$. Neutrophils are part of the first line of defence against invading threats of an infectious nature (25); an increased circulating number following exercise may hence represent a mechanism by which non-specific immunity mechanisms are enhanced (15).This theory is supported by the finding of an increased infection risk in the presence of a reduced number of neutrophils in the 
circulation, as found for example as a result of auto-immune diseases or drugs affecting myeloid precursor cells (17).

As with the relationship between exercise and infection risk, exercise intensity appears to be an important mediator of changes in neutrophil numbers and activation by directly affecting modulators such as plasma epinephrine, cortisol, or cytokines $(15,17,21,25)$. This may further impact on the expression of neutrophil surface receptors, such as the interleukin-8 (IL-8) receptor (CXCR2 - involved in neutrophil chemotaxis) or P-selectin glycoprotein ligand-1 (PSGL-1 - promoting neutrophil tethering to endothelial cells) (8) which may also play a crucial role in the appearance of neutrophils in the circulation and their migration and cytotoxic capacity. Given the central role of exercise intensity in mounting neutrophil responses we propose to elucidate whether upper body exercise has a similar capacity to induce these responses as lower body exercise. Hence, the aim of this study was to compare the neutrophil response of arm exercise and cycling, which were matched for (1) relative and (2) absolute intensities.

\section{MATERIALS AND METHODS}

Blood samples were obtained as part of a study published previously (14). As a consequence, the exercise intervention, participant and exercise characteristics are identical between those studies. The study was approved by the University’s Ethics committee.

\subsection{Participants and experimental design}

Twelve recreationally trained male individuals volunteered to participate (age: $25 \pm 4$ years, body mass: $76 \pm 9 \mathrm{~kg}$, sporting activity $4.0 \pm 1.2 \mathrm{~h} \cdot$ week $^{-1}$, $\mathrm{V}_{2 \text { peak arms }}: 32.1 \pm 6.0 \mathrm{~mL} \cdot \mathrm{kg}^{-}$ 
$\left.{ }^{1} \cdot \mathrm{min}^{-1}, \dot{\mathrm{V}} \mathrm{O}_{\text {2peak legs }}: 46.2 \pm 6.8 \mathrm{~mL} \cdot \mathrm{kg}^{-1} \cdot \mathrm{min}^{-1}\right)$. They visited the laboratory on five occasions for two preliminary and three main trials. Initially, body mass and height were determined using scales (model 770, seca, Birmingham, UK) and a Leicester height measure (seca, Birmingham, UK). In the two preliminary trials (visits 1 and 2), peak oxygen consumption $\left(\dot{\mathrm{VO}}_{2 \text { peak }}\right.$ ) was determined during arm exercise (determining $\dot{\mathrm{V}} \mathrm{O}_{\text {2peak arms }}$ ) using an arm crank ergometer (Angio, Lode, Groningen, Holland) or during cycling exercise (determining $\dot{\mathrm{V}} \mathrm{O}_{2 \text { peak legs }}$ ) using a cycle ergometer (Excalibur, Lode Groningen, Holland) in a randomised order. For this, participants performed a graded exercise test to exhaustion, with an initial power output of $35 \mathrm{~W}$ (arms) and $70 \mathrm{~W}$ (legs), respectively; power output was then increased every three minutes by $15 \mathrm{~W}$ (arms) or $30 \mathrm{~W}$ (legs) until exhaustion. Arm exercise was performed in a sitting position, the centre of the crank at shoulder level with arms slightly flexed at maximum reach, cycling with legs slightly flexed at maximum reach. Settings were noted and replicated for all main trials.

Main trials were performed in a randomised order after a $24 \mathrm{~h}$ food standardisation period without caffeine and with no exercise allowed $24 \mathrm{~h}$ before the experiments. To account for diurnal variations exercise tests were performed in the morning (start: 07:45-09:15) for all participants and at the same time of day for each individual participant. The three main trials

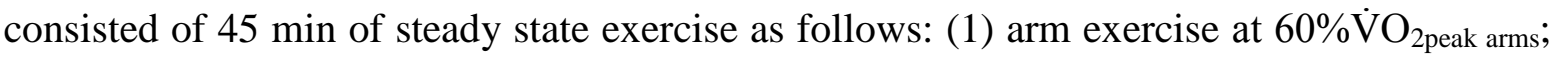

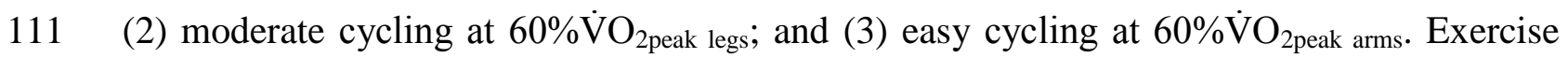

112 intensities and duration were chosen in accordance with exercise prescription guidelines for 113 moderately trained individuals (1). A five minute warm-up was performed at 50\% of the 114 start load before each condition. Oxygen uptake was determined in five minute intervals and 115 power output was adjusted if necessary. For all experiments, oxygen uptake was determined 116 using Douglas bags and a gas analyser (Servomex 1440, Servomex Ltd, Crowborough, UK), 
117 and heart rate was continuously monitored using a Polar RS400 (POLAR, Kempele,

118 Finland) monitor. Heart rate data collected in the first 10 minutes of the main trial were

119 excluded from analysis to avoid inclusion of the reduced values at the beginning of exercise.

120 Participants further indicated their rating of perceived exertion (RPE) on a scale ranging

121 from 6 to 20 (2). Water during exercise was given ad libitum, with the help of a straw to

122 allow hand free water intake, particularly important for the arm cranking modality. Water

123 intake in the post-exercise period was recorded and replicated for the remaining main trials;

124 food and other drinks than water were not allowed during the main trials. Ten participants

125 were invited to the laboratory for a $4^{\text {th }}$ main trial, which consisted of a 45 min rest period

126 instead of the exercise intervention to carry out flow cytometric analyses at rest.

\section{$127 \quad 2.2 \quad$ Blood collection}

128 Participants were lying in a supine position for venous blood sample collection. Blood was

129 collected into $\mathrm{K}_{3}$ EDTA (for hematology and plasma marker analysis) and heparin (for

130 neutrophil degranulation and flow cytometric analysis) containers from a superficial arm

131 vein by venepuncture. Collection times were before, immediately after, and at $2 \mathrm{~h}$ and $4 \mathrm{~h}$

132 after exercise, timings being the same for those participants performing the rest trial. Apart

133 from the collection immediately after exercise, participants rested on a bed for 10 minutes 134 before the blood sample was taken.

\section{$135 \quad 2.3 \quad$ Hematology}

136 Leukocyte subtype numbers, hemoglobin and the hematocrit in whole blood were

137 determined immediately after collection using an automated hematology analyser (Coulter

138 Ac·T 5diff OV; Beckman Coulter, High Wycombe, UK). Blood volume changes were 
139 estimated from hemoglobin values (7), and leukocyte subset numbers corrected for changes

140 in blood volume.

\section{$141 \quad 2.4 \quad$ Plasma markers}

142 Following centrifugation $\left(10 \mathrm{~min}\right.$ at $350 \mathrm{~g}$ and $\left.4^{\circ} \mathrm{C}\right)$ plasma was stored at $-20{ }^{\circ} \mathrm{C}$ until 143 analysis. Interleukin-8 (R\&D systems, Minneapolis, US), epinephrine (IBL International

144 GmBH, Hamburg, Germany) and cortisol (DRG Instruments GmbH, Marburg, Germany)

145 were determined by enzyme-linked immunosorbent assay (ELISA). The within assay co146 efficient of variation for this analysis performed was $5.6 \pm 4.2 \%$ for IL-8, $4.1 \pm 3.9 \%$ for

147 epinephrine and $2.7 \pm 2.7 \%$ for cortisol. As the focus of this study was on plasma marker 148 concentration affecting leukocytes and other effectors rather than determining the fold 149 change of plasma marker production, plasma concentration was not corrected for plasma 150 volume changes.

\subsection{Flow cytometry}

152 The following fluorochromes were used in this study: FITC-conjugated CD182 (also known as CXCR2, the IL-8 receptor), FITC-conjugated IgG1, $\kappa$ isotype control, PE-conjugated

154 CD16, APC-conjugated IgG2a, $\kappa$ isotype control (BD, Oxford, UK), and APC-conjugated

155 CD162 (also known as PSGL-1) (eBioscience, Hatfield, UK). Within 2 h of sample 156 collection whole blood $(120 \mu \mathrm{L})$ was incubated with the above fluorochromes in duplicate:

157 (1) CD182, CD16, CD162 (2) FITC isotype control, CD16, APC isotype control. Labelling 158 was carried out on ice for 20 min, followed by lysis with FACS lysis buffer (BD, Oxford, $159 \mathrm{UK}$ ) and incubation in the dark for another $10 \mathrm{~min}$. Samples were then centrifuged for 6 min 160 at $440 \mathrm{~g}$, the supernatant was removed and the cell pellet re-suspended with $1.5 \mathrm{~mL}$ ice-cold 
161

162

163

164

165

166

167

168

169

170

171

172

173

174

175

176

177

178

179

180

PBS. The centrifugation and supernatant removal steps were repeated, and the cell pellet was re-suspended in $400 \mu \mathrm{L}$ ice-cold PBS for immediate analysis with the flow cytometer (FACSCalibur equipped with the CellQuest software package, BD Biosciences, Oxford, UK), collecting 100,000 events per sample. All analyses were performed for neutrophils, defined as the CD16+ cells. Histogram plots of CD16+ cells incubated with the respective isotype control antibodies alone were used to define the threshold of positive staining for CD182 and CD162 respectively. This threshold was then used to determine the percentage of CD16+ cells expressing CD182 and CD162, respectively, and the geometric mean of fluorescence intensity (GMFI) was determined for CD182 and CD162, respectively.

\subsection{Neutrophil degranulation}

Under sterile conditions, $1 \mathrm{~mL}$ of whole blood was incubated with $50 \mu \mathrm{L}$ of bacterial stimulant (84015; Sigma-Aldrich, Dorset, UK) or a control medium (50 $\mu \mathrm{L}$ RPMI 1640, Sigma-Aldrich, Dorset, UK) for $60 \mathrm{~min}$ at $37^{\circ} \mathrm{C}$; samples were gently inverted before incubation and at $30 \mathrm{~min}$. Following incubation, samples were centrifuged and the plasma stored at $-20^{\circ} \mathrm{C}$. Plasma elastase concentration was then determined in duplicate by ELISA (2BScientific, Upper Heyford, UK) for the determination of neutrophil degranulation activity, the within assay co-efficient of variation being $2.2 \pm 1.9 \%$. The final elastase concentration was corrected for changes in plasma volumes. Net elastase release was calculated by subtracting the stimulated from the unstimulated value, and the elastase release per neutrophil was calculated by dividing this number by the neutrophil number. 
182 The SPSS 23.0 statistical package (SPSS Inc., Chicago IL, USA) was used for all statistical 183 analyses. Means and standard deviations were computed for all variables, and normality was 184 checked with the Shapiro Wilk test. Non-normal data were converted using square root (for 185 CXCR2 GMFI) or logarithmic (for neutrophil number) transformations to achieve normality. A repeated measures two-way (exercise trial, time) analysis of variance (ANOVA) was conducted on normally distributed blood derived variables. Data showing significant interaction effects were further analysed with repeated measures ANOVAs,

189 focussing on time points standing out following visual inspection of plotted data. Huynh-

190 Feldt corrections were applied when sphericity was violated and Sidak adjustments applied for post-hoc comparisons. Non-normal data that were impossible to convert to achieve normality were analysed using repeated, Bonferroni corrected Wilcoxon signed rank tests. Physiological exercise descriptors were analysed using a one-way (exercise trial) repeated

194 measures ANOVA or the non-parametric equivalents for non-normal and RPE data. For

195 main trials, Pearson's correlation coefficients (R) and 2-tailed significances were computed 196 for the bivariate relationsships between neutrophil number and the independent variables 197 heart rate, epinephrine and IL-8. Statistical significance was accepted at $\mathrm{P}<0.05$.

\section{$198 \quad 3 \quad$ RESULTS}

\subsection{Exercise responses}

The exercise trials resulted in distinctively different physiological and psychophysiological responses, the lowest power output, heart rate and RPE values found for easy cycling. Arm

202 exercise and easy cycling did not differ with regards to absolute oxygen uptake; arm 
exercise and moderate cycling did not differ with regards to relative oxygen uptake (Table I).

\subsection{Neutrophil and plasma markers}

206

Neutrophil numbers were increased following all exercise trials but also following the rest trial (main effect of time: $\mathrm{P}<0.001$ ), with a time $\mathrm{x}$ trial interaction indicating a blunted response for the rest and easy cycling trial $(\mathrm{P}<0.001)$. Arm exercise induced a larger neutrophil number increase in the recovery period when compared with easy cycling $(\mathrm{P}=$ 0.009), but not when compared with moderate cycling $(\mathrm{P}=0.90)$. Neutrophil degranulation, expressed as elastase secretion per neutrophil cell, was significantly reduced at $2 \mathrm{~h}$ post exercise (effect of time: $\mathrm{P}<0.02$, effect of condition: $\mathrm{P}=0.53$, condition $\mathrm{x}$ time interaction: $\mathrm{P}=0.14$; Figure 1).

The plasma epinephrine concentration increased from pre to post exercise for all exercise trials $(\mathrm{P}<0.001)$, but the post-exercise epinephrine concentration was higher for arm exercise than for easy cycling $(\mathrm{P}=0.02)$. The plasma cortisol concentration was lower in the recovery period for all exercise trials $(\mathrm{P}<0.05)$, with no difference between trials. The IL-8 plasma concentration increased from pre to post $(\mathrm{P}=0.01)$, but no difference between exercise trials was found for IL-8 ( $\mathrm{P}=0.80$; Figure 2).

Even though some of the analysed regressions explained up to $52 \%\left(\mathrm{R}^{2}\right)$ of the random variation between the dependent variable neutrophil number and the independent variables heart rate, epinephrine and IL-8, these relationships were largely found to be insignificant (Table II). The dependent variable consistently associated with positive relationships was the neutrophil number $2 \mathrm{~h}$ post exercise. 
The CXCR2 and CD162 GMFI showed circadian rhythms, with the lowest values found $2 \mathrm{~h}$

and $4 \mathrm{~h}$ after the exercise trials but also the rest trial $(\mathrm{P}<0.001)$. No differences were found between the three exercise trials and the rest trial $(\mathrm{P}>0.55)$. The percentage of neutrophils expressing CXCR2 $2 \mathrm{~h}$ post exercise was reduced for all exercise trials when compared with the rest trial $(\mathrm{P}<0.05)$, but the exercise trials did not differ between each other $(\mathrm{P}=0.17)$. The percentage of neutrophils expressing CD162 did not change over time for any trial (P > 0.05; Figure 3).

\section{DISCUSSION}

This is the first study to compare the neutrophil response in intensity matched trials between arm and cycling exercise. It provides further evidence with regards to the exercise modality specific potential of mounting an acute neutrophil response, which is of practical importance for those restricted to upper body exercise. The main finding of this study was that arm exercise and cycling at the same relative exercise intensity induces a comparable acute neutrophil recruitment into the circulation. On the other hand, cycling at the same absolute oxygen uptake as arm exercise results in a blunted response for cycling. Lower responses for easy cycling were further observed for the plasma epinephrine concentration, heart rate, and RPE. Arm exercise also resulted in the same decline of neutrophil degranulation in the recovery period as leg exercise. It therefore appears that the arm cranking modality has the capacity to induce the same acute neutrophil response as the cycling modality.

A second finding was the presence of circadian rhythms for neutrophil markers: a decline in the GMFI of the neutrophil surface markers CXCR2 and PSGL-1 was observed over the $5 \mathrm{~h}$ study period for all exercise and rest conditions. 


\section{$4.1 \quad$ Neutrophil responses}

252

253

254

255

256

257

258

259

260

261

262

263

264

265

266

267

268

269

270

271

272

273

\subsubsection{Neutrophilia}

The lowest epinephrine concentrations post exercise were found for easy cycling, the trial where the neutrophil response was blunted, corroborating earlier data pointing out the importance of relative exercise intensity in this response $(15,26)$. It has been suggested levels of epinephrine may impact on the neutrophil response through direct mechanisms, such as epinephrine dependent recruitment of leukocyte subgroups into the circulation (22, 30). Patterns of leukocyte mobilisation similar to the current study have been found when modulating core temperature or exercise intensity, with the modes inducing the most pronounced epinephrine response resulting in the largest increase in neutrophil numbers (27) or cytokine secretion $(21,23)$. However, neutrophil numbers have been shown not to be affected by epinephrine infusion mimicking the epinephrine increase during exercise (31). Further, it has been shown that epinephrine increases during non-exercise based interventions (hot water immersion) alone are not sufficient to induce neutrophilia as observed during exercise, despite comparable epinephrine levels (12). The results of the present study corroborate these findings: the insignificant correlations between epinephrine concentration post exercise and neutrophil numbers both post $(\mathrm{R} \leq 0.13)$ and $2 \mathrm{~h}$ post exercise $(\mathrm{R} \leq 0.28)$ imply that epinephrine contributes, if at all, only marginally to exercise induced neutrophilia. A direct causal relationship between the observed larger increases in epinephrine and neutrophil numbers in the arm exercise trial compared to the absolute intensity matched cycling trial is therefore unlikely, and other factors are likely to contribute to this. 
274 Such factors may include neutrophil surface receptors. Given their roles in IL-8 mediated

275

276

277 chemotaxis (CXCR2) and tethering to endothelial cells (PSGL-1) it could be hypothesised that these receptors play a role in exercise induced neutrophilia. Indeed, the present results do provide support for a mechanistic role of CXCR2, but not PSGL-1, in this process. Even though exercise is unable to alter the natural decrease of the GMFI over time, a reduction in CXCR2 expression in the exercise trials can be observed $2 \mathrm{~h}$ post exercise, which is different from the resting condition. In the context of neutrophilia it could be argued that a reduced expression of CXCR2 allows more neutrophils to remain in the circulation as their IL-8 dependent migration capacity into the extravascular compartment is reduced. This is in line with the observation that intravenous administration of IL-8 inhibits neutrophil accumulation to intradermal sites of inflammation, possibly due to the disruption of concentration gradients (16). The correlations between IL-8 and neutrophil number found in the present study further support this concept: even tough not significant for all trials, the positive relationship between IL-8 concentration and neutrophil numbers $2 \mathrm{~h}$ post exercise imply that central elevations of IL-8 may help inducing neutrophilia.

Further to this, it has been postulated that the shedding of receptors may serve as an adaptive mechanism for inhibiting excessive inflammatory reactions (21) which may arise given the increased numbers of neutrophils in the circulation. Similarly to CXCR2, other surface markers associated with neutrophil adhesion, such as CD11b (21) or L-selectin (32), have been shown to be downregulated in response to moderate and high intensity exercise. It is important to know, though, that these findings are based on research designs that did not feature a resting control trial - given the findings of the present study a potentially crucial omission given the circadian effects shown for CXCR2 and PSGL-1. 
No changes in cortisol were found between trials. Previous evidence suggests that exercise intensities above $\sim 60 \% \mathrm{VO}_{2 \max }$ can increase cortisol plasma concentrations, whereas intensities below $\sim 50 \% \mathrm{VO}_{2 \max }$ can result in a decrease, as elimination rates during low intensity exercise are higher than at rest (15). It is therefore possible that the exercise 303 intensities chosen in the current study were not high enough to trigger a cortisol response.

304 Changes in cortisol concentration are hence unlikely to be the main mechanism to govern 305 the neutrophilia observed given the fact that the more strenuous activities resulted in pronounced differences in neutrophilia but no difference in the cortisol response. However, in line with observations in lymphocytes $(4,15)$ the general decline in cortisol over time coincided with the general increase in neutrophil numbers; importantly, a small degree of neutrophilia was also observed in the rest condition. The reduction in cortisol with its general anti-inflammatory properties may therefore aid the development of neutrophilia over

311 the course of the day, strengthening the first line of defence when the likelihood of infection 312 is increased due to an increased exposure to pathogens.

314 Further to the discussed plasma and neutrophil markers that are potentially involved in 315 neutrophilia (epinephrine, cortisol, IL-8, or neutrophil receptors), the demargination of neutrophils via the increase in cardiac output (12), increased blood flow through viscera and muscles and a "general" sympathetic activation (15) have been suggested to induce a rise in 318 neutrophil number in the circulation. Cardiac output was not directly assessed in the present 319 study; however, heart rate was significantly higher for arm exercise when compared with the easy cycling trial, and correlations between heart rate and neutrophil number were positive. In fact, heart rate was the only variable with $\mathrm{R}>0.50$ for all trials when related to neutrophil 322 number $2 \mathrm{~h}$ post exercise. Whilst we appreciate that heart rate is only an approximation of 323 cardiac output, these data support the notion of cardiac output as an important factor to 
324 induce neutrophila (12). It is also worth noting that the increase in neutrophil numbers immediately following exercise did not correlate well with any of the presently analysed predictor variables, implying that the effects of adrenaline, IL-8 and heart rate take time (two hours) to manifest in elevated neutrophil numbers. This is in contrast to earlier suggestions that attributed the initial neutrophilia following exercise to factors such as cardiac output and epinephrine, whilst the mechanism underlying delayed neutrophilia following exercise has been suggested to involve glucocorticoids (15).

On a final note, differences in sympathetic activation were also reflected in the RPE responses, lowest for the easy cycling modality. Intensity differences between exercise trials may further explain differences in neutrophilia through their impact on a broader range of markers than catecholamines and glucocorticoids, such as a host of interleukins $(14,23,29)$ which ultimately affect neutrophil behaviour (8).

\subsubsection{Neutrophil degranulation}

In agreement with the present study, similar reductions in bacteria-stimulated neutrophil degranulation, a marker of the reactivity of an individual cell against pathogens, have been reported following exercise $(5,6,12,22,28)$. This decrease in neutrophil function following aerobic exercise has also been observed when investigating migratory capacity (9, 33). It has been found earlier that exercise and rises in cortisol can induce the release of immature neutrophils from the bone marrow into the circulation $(15,22)$. As they contain less granular digestive enzymes this may be a mechanism by which exercise reduces average phagocytic activity of the neutrophil population as a whole (22). The present study shows that increases in cortisol are not a requirement to reduce in bacteria-stimulated neutrophil degranulation. In addition, in contrast to previous studies (22) the suppression of neutrophil degranulation 
observed in the present study appears to be mode and intensity independent - however, this

349 is likely the result of insufficient statistical power to analyse this detail, as a trend for a reduced depression was seen for easy cycling.

\subsection{Future directions}

The current study shows rises in neutrophil numbers that are dependent on relative exercise

353 intensity. For future research, it would be of interest to investigate neutrophil subpopulations

354 to assess the origin of the neutrophils released into the circulation following exercise. For 355 this, their maturity could be assessed by morphology (e.g., band vs segmented neutrophils). 356 Another interesting field of research is the investigation of markers involved in neutrophil 357 mobilisation. Whilst we have measured the plasma concentration of the chemokine IL-8, 358 granulocyte colony stimulating factor (G-CSF) has been shown to increase in response to 359 exercise that induces neutrophilia (12) and may explain some of the exercise intensity 360 dependent differences found in the present study. Finally, we have focussed on elastase as a marker for neutrophil degranulation, and it may be of interest to investigate other makers of 362 neutrophil degranulation such as myeloperoxidase (20).

\section{CONCLUSION}

364 Arm and leg exercise elicits the same neutrophil response when performed at the same relative intensity, cycling at the same absolute intensity as arm exercise results in a blunted increase in neutrophil numbers, heart rate and epinephrine. Populations restricted to arm exercise therefore have a similar capacity to induce a neutrophil response through exercise, which may play a role in host protection following exercise. Heart rate as an integrated measure of exercise stress / cardiac output was the best predictor of neutrophil numbers $2 \mathrm{~h}$ 
370 post for all exercise trials. This study also shows that CXCR2 may be implicated in 371 exercise-induced neutrophilia. Further, the results demonstrate that circadian effects must be 372 considered for neutrophil surface markers enforcing the need for resting control conditions 373 in studies investigating the first line of defence in the circulation.

\section{CONFLICT OF INTEREST}

375 The authors declare no conflict of interest.

\section{$376 \quad 7 \quad$ ACKNOWLEDGEMENTS}

377 To all participants we are thankful for their time and willingness to participate in this study.

378 Thanks are extended to Ms Hannah Carey, Mr Oliver Hooper and Mr U-Peng Tan who 379 assisted in data collection. This research was supported by the Peter Harrison Centre for 380 Disability Sport and a grant from JMP Holdings (Kuala Lumpur, Malaysia). The results of 381 the study are presented clearly, honestly, and without fabrication, falsification, or 382 inappropriate data manipulation, and the results do not constitute endorsement by ACSM. 


\section{FIGURE CAPTIONS}

385 FIGURE 1 - Neutrophil number and neutrophil elastase secretion in response to steady 386 state exercise (mean $\pm \mathrm{SD})$. Significant differences $(\mathrm{P}<0.05)$ : a, different from pre (all 387 trials); b, different from post (all trials); c, different from all time points (all trials); d, 388 difference between arm and leg easy trial.

389

390 FIGURE 2 - Plasma epinephrine, cortisol and interleukin-8 concentrations in response to 391 steady state exercise (mean $\pm \mathrm{SD}$ ). Significant differences $(\mathrm{P}<0.05)$ : a, different from pre 392 (all trials); b, different from post (all trials); c, difference between arm and leg easy trial.

393

394 FIGURE 3 - Neutrophil surface markers in response to steady state exercise (mean \pm SD).

395 GMFI, geometric mean of fluorescence intensity. Significant differences $(\mathrm{P}<0.05)$ : a, 396 different from pre (all trials); b, different from post (all trials); c, all exercise trials different 397 from rest.

398

\section{TABLE CAPTIONS}

400 Table I - Physiological and psychophysiological exercise descriptors.

401 Table II - Bivariate correlations of epinephrine, IL-8, heart rate and neutrophil number. 
403 1. American College of Sports Medicine. ACSM's Guidelines for exercise testing and 404 prescription. 7th ed. (Philadelphia): Lippincott Williams \& Wilkins; 2006.

405 2. Borg GA. Psychophysical bases of perceived exertion. Med Sci Sports Exerc. 406 1982;14(5):377-81.

407 3. Centers for Disease Control and Prevention Web site [Internet]. National Health 408 Interview Survey; [cited 2016 Dec 15]. Available from: 409 https://www.cdc.gov/nchs/nhis/ad292tb1.htm.

410 4. Cupps TR, Fauci AS. Corticosteroid-mediated immunoregulation in man. Immunol Rev. $411 \quad$ 1982;65:133-55.

412 5. Davison G. Innate immune responses to a single session of sprint interval training. Appl 413 Physiol Nutr Metab. 2011;36(3):395-404.

414 6. Davison G, Gleeson $\mathrm{M}$. The effect of 2 weeks vitamin $\mathrm{C}$ supplementation on 415 immunoendocrine responses to $2.5 \mathrm{~h}$ cycling exercise in man. Eur J Appl Physiol. 416 2006;97(4):454-61.

417 7. Dill DB, Costill DL. Calculation of percentage changes in volumes of blood, plasma, and 418 red cells in dehydration. J Appl Physiol. 1974;37(2):247-8.

419 8. Futosi K, Fodor S, Mocsai A. Neutrophil cell surface receptors and their intracellular 420 signal transduction pathways. Int Immunopharmacol. 2013;17(3):638-50.

421 9. Gavrieli R, Ashlagi-Amiri T, Eliakim A et al. The effect of aerobic exercise on neutrophil 422 functions. Med Sci Sports Exerc. 2008;40(9):1623-8. 
423 10. Helge JW, Klein DK, Andersen TM et al. Interleukin-6 release is higher across arm than

424 leg muscles during whole-body exercise. Exp Physiol. 2011;96(6):590-8.

425 11. Kouda K, Furusawa K, Sugiyama H et al. Does 20-min arm crank ergometer exercise 426 increase plasma interleukin-6 in individuals with cervical spinal cord injury? Eur J Appl $427 \quad$ Physiol. 2012;112(2):597-604.

428 12. Laing SJ, Jackson AR, Walters R et al. Human blood neutrophil responses to prolonged 429 exercise with and without a thermal clamp. J Appl Physiol. 2008;104(1):20-6.

430 13. Leicht CA, Bishop NC, Goosey-Tolfrey VL. Mucosal immune responses to treadmill 431 exercise in elite wheelchair athletes. Med Sci Sports Exerc. 2011;43(8):1414-21.

432 14. Leicht CA, Paulson TAW, Goosey-Tolfrey VL, Bishop NC. Arm and intensity-matched 433 leg exercise induce similar inflammatory responses. Med Sci Sports Exerc. 434 2016;48(6):1161-8.

435 15. McCarthy DA, Dale MM. The leucocytosis of exercise. A review and model. Sports 436 Med. 1988;6(6):333-63.

437 16. Mukaida N, Harada A, Matsushima K. Interleukin-8 (IL-8) and monocyte chemotactic 438 and activating factor (MCAF/MCP-1), chemokines essentially involved in inflammatory and 439 immune reactions. Cytokine Growth Factor Rev. 1998;9(1):9-23.

440 17. Nagatomi R. The implication of alterations in leukocyte subset counts on immune 441 function. Exerc Immunol Rev. 2006;12:54-71. 
18. National Health Service (NHS) England Web site [Internet]. Improving Wheelchair

443

services; $\quad$ [cited

2016

Dec

15].

Available

from:

444 https://www.england.nhs.uk/ourwork/pe/wheelchair-services.

445 19. Nieman DC. Exercise, infection, and immunity. Int J Sports Med. 1994;15 Suppl $446 \quad 3: S 131-41$.

447 20. Peake J, Peiffer JJ, Abbiss CR et al. Body temperature and its effect on leukocyte 448 mobilization, cytokines and markers of neutrophil activation during and after exercise. Eur $J$ Appl Physiol. 2008;102(4):391-401.

450

21. Peake J, Wilson G, Hordern $\mathrm{M}$ et al. Changes in neutrophil surface receptor expression, 451 degranulation, and respiratory burst activity after moderate- and high-intensity exercise. $J$ 452 Appl Physiol. 2004;97(2):612-8.

453 22. Peake JM. Exercise-induced alterations in neutrophil degranulation and respiratory burst 454 activity: possible mechanisms of action. Exerc Immunol Rev. 2002;8:49-100.

455 23. Peake JM, Suzuki K, Hordern M, Wilson G, Nosaka K, Coombes JS. Plasma cytokine 456 changes in relation to exercise intensity and muscle damage. Eur J Appl Physiol. 2005;95(5457 6):514-21.

24. Peake JM, Suzuki K, Wilson G et al. Exercise-induced muscle damage, plasma 459 cytokines, and markers of neutrophil activation. Med Sci Sports Exerc. 2005;37(5):737-45.

25. Pyne DB. Regulation of neutrophil function during exercise. Sports Med. 1994;17(4):245-58. 
26. Quindry JC, Stone WL, King J, Broeder CE. The effects of acute exercise on neutrophils and plasma oxidative stress. Med Sci Sports Exerc. 2003;35(7):1139-45.

464

465

466

467

468

469

470

471

472

473

474

475

476

477

478

479

480

481

27. Rhind SG, Gannon GA, Shek PN et al. Contribution of exertional hyperthermia to sympathoadrenal-mediated lymphocyte subset redistribution. $J$ Appl Physiol. 1999;87(3):1178-85.

28. Robson PJ, Blannin AK, Walsh NP, Castell LM, Gleeson M. Effects of exercise intensity, duration and recovery on in vitro neutrophil function in male athletes. Int $J$ Sports Med. 1999;20(2):128-35.

29. Scott JP, Sale C, Greeves JP, Casey A, Dutton J, Fraser WD. Effect of exercise intensity on the cytokine response to an acute bout of running. Med Sci Sports Exerc. 2011;43(12):2297-306.

30. Starkie RL, Rolland J, Febbraio MA. Effect of adrenergic blockade on lymphocyte cytokine production at rest and during exercise. Am J Physiol Cell Physiol. 2001;281(4):C1233-40.

31. Steensberg A, Toft AD, Schjerling P, Halkjaer-Kristensen J, Pedersen BK. Plasma interleukin-6 during strenuous exercise: role of epinephrine. Am J Physiol Cell Physiol. 2001;281(3):C1001-4.

32. van Eeden SF, Granton J, Hards JM, Moore B, Hogg JC. Expression of the cell adhesion molecules on leukocytes that demarginate during acute maximal exercise. J Appl Physiol. 1999;86(3):970-6. 
482 33. Wolach B, Gavrieli R, Ben-Dror SG, Zigel L, Eliakim A, Falk B. Transient decrease of 483 neutrophil chemotaxis following aerobic exercise. Med Sci Sports Exerc. 2005;37(6):94948454.

485 
TABLE I. Physiological and psychophysiological exercise descriptors.

\begin{tabular}{|c|c|c|c|}
\hline \multirow[t]{2}{*}{ Parameter } & \multicolumn{3}{|c|}{ Exercise mode } \\
\hline & Arm exercise & Moderate cycling & Easy cycling \\
\hline Power output [W] & $73 \pm 14^{*}$ & $148 \pm 30^{*}$ & $101 \pm 25^{*}$ \\
\hline Heart rate $\left[\mathrm{b} \cdot \mathrm{min}^{-1}\right]$ & $141 \pm 9^{*}$ & $150 \pm 12^{*}$ & $123 \pm 11^{*}$ \\
\hline Average RPE & $13.3(12.6,13.9)$ & $13.2(12.7,14.0)$ & $10.6(10.0,11.0)^{*}$ \\
\hline$\dot{\mathrm{VO}_{2}}\left[\mathrm{~L} \cdot \mathrm{min}^{-1}\right]$ & $1.50 \pm 0.28$ & $2.16 \pm 0.34^{*}$ & $1.50 \pm 0.28$ \\
\hline$\% \dot{\mathrm{V} O}{ }_{2 \text { peak arms }}$ & $62.3 \pm 1.4$ & - & $62.3 \pm 1.1$ \\
\hline$\% \dot{\mathrm{V}} \mathrm{O}_{2 \text { peak cycling }}$ & - & $62.3 \pm 1.0$ & $43.2 \pm 5.4 *$ \\
\hline
\end{tabular}

RPE, rating of perceived exertion. Data indicate mean \pm SD or median (lower quartile, upper quartile). *Significant difference to both other modalities $(\mathrm{P}<0.05)$ 
Table II - Bivariate correlations of epinephrine, interleukin-8, heart rate and neutrophil number.

\begin{tabular}{llll}
\hline Comparison & & \multicolumn{1}{c}{ Exercise mode } & \\
& Arm exercise & Moderate cycling & Easy cycling \\
\hline $\begin{array}{l}\text { Epinephrine post vs } \\
\text { neutrophil number post }\end{array}$ & $\mathrm{R}=-0.16$ & $\mathrm{R}=0.09$ & $\mathrm{R}=0.13$ \\
& $\mathrm{P}=0.63$ & $\mathrm{P}=0.78$ & $\mathrm{P}=0.68$ \\
Epinephrine post vs & $\mathrm{R}=0.21$ & $\mathrm{R}=0.24$ & $\mathrm{R}=0.34$ \\
neutrophil number 2h post & $\mathrm{P}=0.52$ & $\mathrm{P}=0.45$ & $\mathrm{P}=0.28$ \\
$\begin{array}{l}\text { IL-8 post vs } \\
\text { neutrophil number post }\end{array}$ & $\mathrm{R}=-0.15$ & $\mathrm{R}=-0.44$ & $\mathrm{R}=0.08$ \\
& $\mathrm{P}=0.65$ & $\mathrm{P}=0.16$ & $\mathrm{P}=0.80$ \\
IL-8 post vs & $\mathrm{R}=0.72$ & $\mathrm{R}=0.14$ & $\mathrm{R}=0.46$ \\
neutrophil number 2h post & $\mathrm{P}=0.008$ & $\mathrm{P}=0.67$ & $\mathrm{P}=0.13$ \\
Heart rate vs & $\mathrm{R}=0.19$ & $\mathrm{R}=0.007$ & $\mathrm{R}=0.10$ \\
neutrophil number post & $\mathrm{P}=0.56$ & $\mathrm{P}=0.98$ & $\mathrm{P}=0.75$ \\
Heart rate vs & $\mathrm{R}=0.51$ & $\mathrm{R}=0.69$ & $\mathrm{R}=0.52$ \\
neutrophil number 2h post & $\mathrm{P}=0.09$ & $\mathrm{P}=0.01$ & $\mathrm{P}=0.09$ \\
\hline
\end{tabular}

The plasma concentrations of epinephrine and interleukin-8 and the average heart rate during trials were used to compute correlations. 
Figure 1

Arm exercise $\square$ Moderate cycling $\square$ Easy cycling Rest
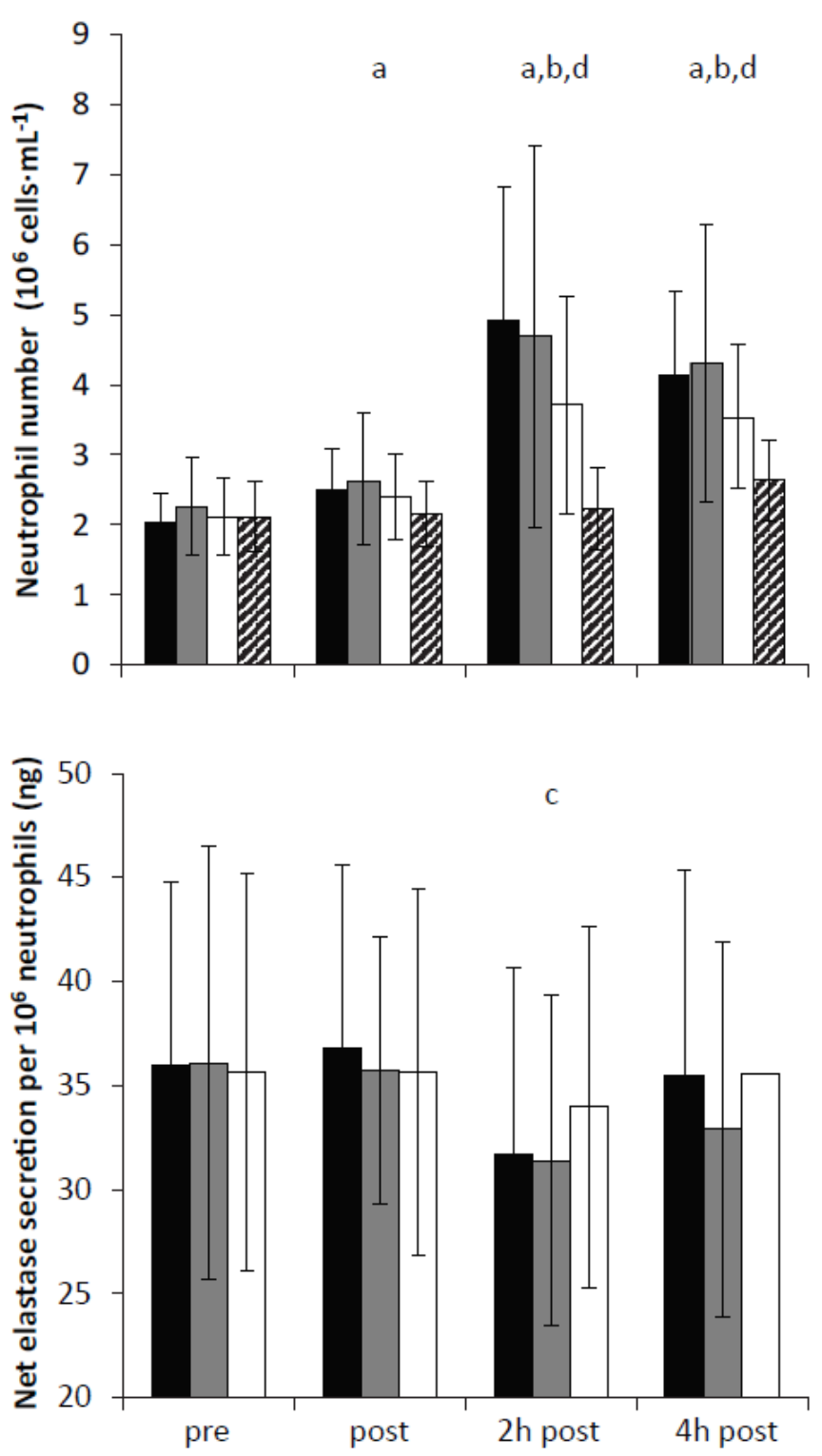

490

491 
Figure 2

- Arm exercise $\square$ Moderate cycling $\square$ Easy cycling
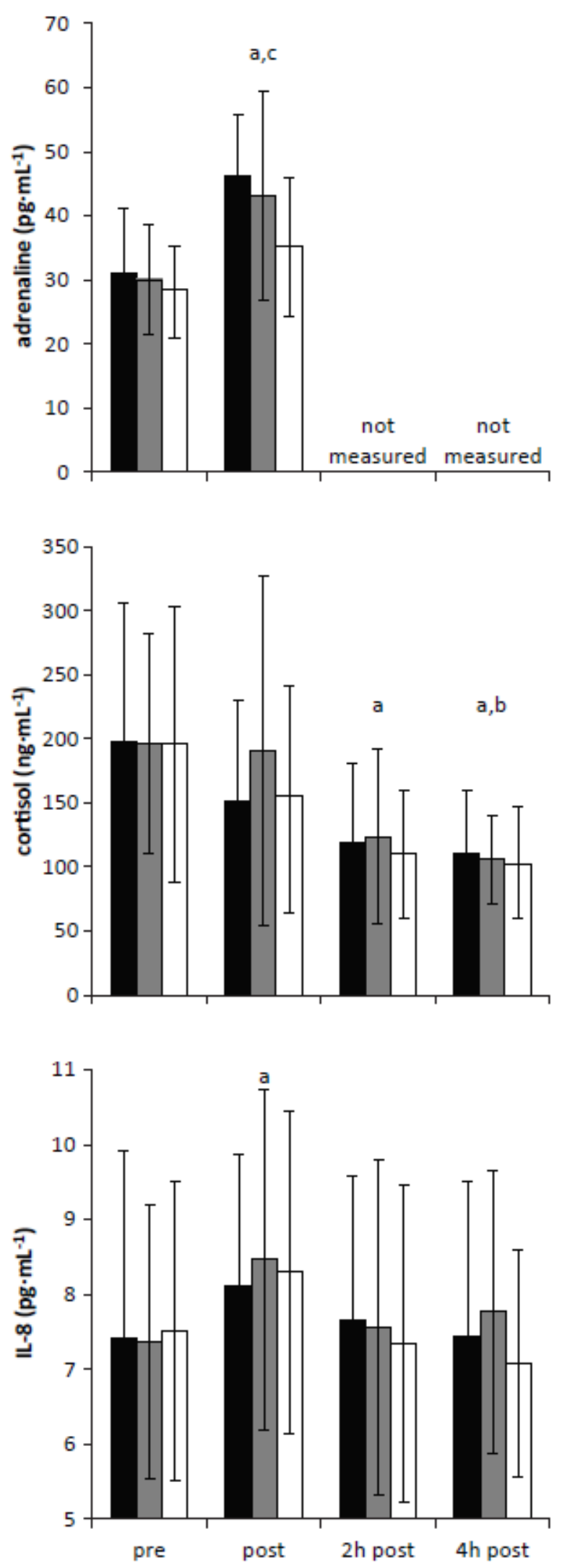
- Arm exercise $\square$ Moderate cycling $\square$ Easy cycling $\boldsymbol{Z}$ Rest
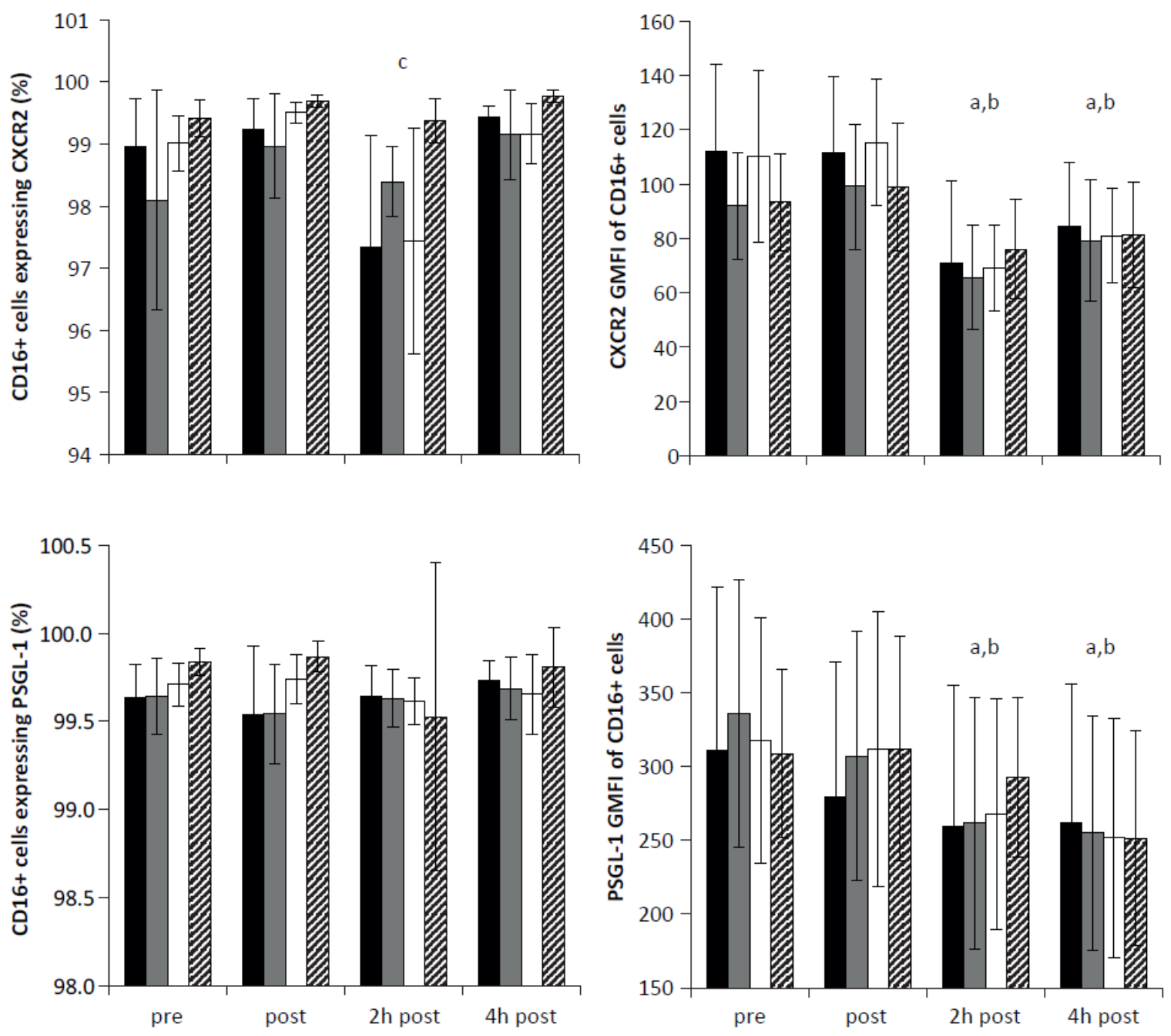